\begin{tabular}{|c|c|}
\hline Chemistry of & $\begin{array}{l}\text { Chem. Met. Alloys } 10 \text { (2017) } 54-60 \\
\text { Ivan Franko National University of Lviv } \\
\text { www.chemetaliourralalor }\end{array}$ \\
\hline
\end{tabular}

\title{
Optical liquid-crystalline nanocomposites based on cadmium caprylate with Au, Ag or $\mathrm{Cu}$ nanoparticles
}

\author{
Vitaliy ASAULA $^{1 *}$, Polina BOROVYK ${ }^{1}$, Galina YAREMCHUK $^{1}$, Tatiana MIRNAYA ${ }^{1}$ \\ ${ }^{1}$ V.I. Vernadskii Institute of General and Inorganic Chemistry of the NAS of Ukraine, \\ Prospect Palladina 32-34, 03680 Kyiv, Ukraine \\ * Corresponding author. Tel.: +380-44-4243211; fax: +380-44-4243070; e-mail: vitaliy.asaula@gmail.com
}

Received May 30, 2017; accepted June 27, 2017; available on-line April 1, 2018

The paper presents experimental results on the synthesis and optical properties of cadmium caprylate nanocomposites with gold, silver or copper nanoparticles. The metal nanoparticles were fabricated by reduction of metal ions directly in a cadmium caprylate liquid-crystalline melt, which performed the function of reductant and stabilizer at the same time. It was found by optical spectroscopy and transmission electron microscopy that the metal nanoparticles had spherical shape and a narrow size distribution range, 15-30 $\mathrm{nm}$ for gold, 14-20 $\mathrm{nm}$ for silver, and 3-7 $\mathrm{nm}$ for copper.

Ionic liquid crystals / Nanoparticles / Noble metals / Optical properties / Plasmon resonance

\section{Introduction}

The interest in the development of methods for the synthesis of nanoparticles of the noble metals gold and silver, as well as copper, and in the study of their properties, is due to their specific physical and chemical properties, which find application in optical, sensor and electronic devices. Determining factors for practical use of the metal nanoparticles are their size, shape and stability. The properties depend first of all on the fabrication method. The creation of optical composites containing metal nanoparticles, and the continuous development of instrumental methods used for their investigation, open up more and more new fields of application for these materials [1-3].

To synthesize and modify optical nanomaterials, various structured media, e.g. glasses [4,5], polymers [6,7] and liquid crystals [8], have been intensively used in recent years. Liquid-crystalline (LC) metal alkanoate melts are of special interest since they permit not only to obtain nanoparticles of defined size and shape, but also to create novel optical and nonlinear optical materials [9].

In this work we have studied the conditions for the synthesis of gold, silver and copper nanoparticles in an ionic liquid - a crystalline matrix of cadmium caprylate $\left(\mathrm{C}_{7} \mathrm{H}_{15} \mathrm{COO}\right)_{2} \mathrm{Cd}$ and binary systems of equimolar composition based on it, Cd, $M / / \mathrm{C}_{8} \mathrm{H}_{15} \mathrm{O}_{2}$, where $M=\mathrm{Li}, \mathrm{Na}, \mathrm{K}, \mathrm{Mg}, \mathrm{Zn}, \mathrm{Pb}-$ and their size and shape. The dependence of the optical absorption of $\mathrm{Au}, \mathrm{Ag}$ and $\mathrm{Cu}$ nanoparticles on experimental conditions such as the synthesis time and temperature, metal-containing precursor concentration, and the (covalent-ionic) nature of the matrix, was established in the work.

\section{Experimental procedure}

Mesogenic metal alkanoates are characterized by a bilayer structure, which is due to a combination of electrostatic ion-ion interactions between metal cations and carboxyl groups and Van der Waals interactions between alkyl chains of the alkanoate anions. They exhibit thermotropic mesomorphism, i.e. form liquid crystals on heating. An example of such an ionic mesogen is cadmium caprylate, which forms a mesophase (smectic A) at $98^{\circ} \mathrm{C}$ and supercools to form a glass at room temperature, which has a smectic bilayer structure [10].

Gold, silver and copper nanoparticles were synthesized in a LC melt of cadmium caprylate $\mathrm{Cd}\left(\mathrm{C}_{8} \mathrm{H}_{15} \mathrm{O}_{2}\right)_{2}$ or binary systems based on this, $\mathrm{Cd}, M / / \mathrm{C}_{8} \mathrm{H}_{15} \mathrm{O}_{2}$, where $M=\mathrm{Li}, \mathrm{Na}, \mathrm{K}, \mathrm{Mg}, \mathrm{Zn}, \mathrm{Pb}$, by reducing precursors of metal ions directly in a molten alkanoate matrix in a temperature range of $100-200^{\circ} \mathrm{C}$ for $3 \mathrm{~h}$.

Hydrogen tetrachloroaurate(III) $\left(\mathrm{H}\left[\mathrm{AuCl}_{4}\right] \times 3 \mathrm{H}_{2} \mathrm{O}\right)$ (r.g., Aldrich) was used as gold ion source. The reaction mixture was homogenized by bubbling it with argon. When the original mixture was loaded into a thermostated oil bath, it had yellowish-white color. During the synthesis the reaction mixture became transparent pinkish. The intensity of the color 
increased during the reaction, and when the reduction was complete, the melt had a saturated purple color. On cooling the melt, mesomorphic cadmium caprylate glasses with gold nanoparticles uniformly distributed throughout the matrix were obtained. The concentration of gold ions varied between 0.25 and 8.0 mol.\%. The mesomorphic glasses based on binary systems of cadmium caprylate with gold nanoparticles had different hues from light pink to claret red.

Silver nanoparticles were synthesized in a cadmium caprylate $\left(\mathrm{Cd}\left(\mathrm{C}_{7} \mathrm{H}_{15} \mathrm{COO}\right)_{2}\right)$ mesophase by reducing the silver ions at the temperature of mesophase existence $\left(98-165^{\circ} \mathrm{C}\right)$ for $3 \mathrm{~h}$. Silver nitrate (r.g., Aldrich) was used for the synthesis. Copper nanoparticles were prepared at $185-200^{\circ} \mathrm{C}$, using copper(I) chloride (r.g.) as copper ion source. On cooling the melts to room temperature, mesomorphic cadmium caprylate glasses with silver or copper nanoparticles were obtained. The glasses had a light brown color, the intensity of which increased with increasing silver or copper nanoparticle content. The concentration of silver and copper ions varied between 0.1 and 0.8 mol.\%. The mesomorphic glasses based on binary systems of cadmium caprylate with silver nanoparticles had different hues from light yellow to dark brown.

The obtained samples were investigated by electron spectroscopy and transmission electron microscopy. The electronic absorption spectra were recorded in the range $250-800 \mathrm{~nm}$ on a Perkin Elmer UV/VIS Lambda 35 spectrophotometer. Quartz cells with a thickness of $40 \mathrm{~nm}$ were used. The morphology of the particles was studied by means of a JEM 100CXII transmission electron microscope $(U=100 \mathrm{kV})$. The texture of the melts and vitrified samples was studied using a polarizing microscope Amplival with hot stage Boemius.

The size of the Au nanoparticles synthesized in the cadmium caprylate matrix was estimated by smallangle X-ray scattering. To carry out the investigation, thin-walled Lindemann capillaries of $0.7 \mathrm{~mm}$ diameter for X-ray analysis were filled with powders of nanocomposites and placed in a furnace with lavsan windows. We used $\mathrm{CuK} \alpha$ radiation, $\mathrm{Ni}$ filter, $U=40 \mathrm{kV}, I=20 \mathrm{~mA}$; the pulse time was $10 \mathrm{~s}$ at angles from $2 \theta=0.06^{\circ}$ and the step $\Delta 2 \theta=0.01^{\circ}$. To process the experimental results, two different ATSAS 2.5 program packages were employed [11].

\section{Results and discussion}

Metal nanoparticles are known to be characterized by strong optical light absorption because of the resonant response of their free electrons to electromagnetic radiation. The nanoparticles of noble metals possess a rare combination of valuable properties: unique characteristics, highly developed surface, high double electrical layer capacity, and nonlinear optical properties [7].
One of the key characteristics of metal nanoparticles, which affects their optical and nonlinear optical properties, is the size distribution. Solving the inverse problem, based on the particle size distribution characteristics the mechanism can be reconstructed [12]. To describe the formation and structuring of metal nanoparticles in condensed media - matrices, some authors (see [12] and references therein) propose a model within the framework of the so-called nonclassical mechanisms of growth of anisotropic nanoparticles, coordinated growth [13] or oriented attachment [14]. The essence of the model consists in the following: At the initial growth stages, small metallic clusters with high reactivity are formed in the condensed medium. As a result of the rapid growth, a sol of nucleating nanoparticles, containing an imperfect metal skeleton and a loose surface layer, is formed. The structure of the metal skeleton has numerous defects and is capable of self-organization leading to crystallization of the interior and segregation of surface layers. In the course of such crystallization-segregation, detachment of cluster fragments from less structured nanoparticles and their transfer through the bulk to another structured nanoparticle may be possible.

From the analysis of literature data [15-17] it follows that the size and degree of structuring of the metal nanoparticles depend on the rate of nucleation and diffusion. For instance, if the diffusion rate of cluster fragments (or atoms) of metal is higher than the nucleation rate, then nanoparticles of smaller size predominate. In the opposite case, large nanoparticles are formed.

The size, morphology, stability, and other characteristics of the synthesized metal nanoparticles, e.g. copper nanoparticles, are influenced by a number of factors, including the nature and concentration of the metal precursor, reductant, and solvent. An important characteristic of the precursors is their redox potential, the value of which is greatly affected by the above factors [18].

Such characteristics of metal nanoparticles as hydrophilicity, the position of the absorption peak of surface plasmon resonance or giant Raman scattering of electromagnetic radiation, depend on the chemical composition and structure of the surface. In a condensed medium there are no "bare" nanoparticles, which have no adsorbed components of the environment. The composition and structure of the medium directly affect the average particle size. The stronger the interaction of the surface atoms with adsorbed components of the condensed phase surrounding the nanoparticle, or the higher the activation barrier of the interactions destroying the structure of the surface layer, the smaller the particle size at which a stable nanostructured system is formed [12]. The interaction between the surface atoms and the environment components competes with the rapid growth of metal nanoparticles. When the conditions for the occurrence of competitive process arise, the 
process mainly takes place in a real system, which gives rise to maximum decrease of the surface energy. According to the energy difference arising from stabilization, the processes can be arranged in the following order: classical interaction $>$ donoracceptor interaction $>$ electrostatic interaction. The formation of covalent bonds between the surface atoms and the functional groups of the modifier results in the formation of stable systems with the smallest average particle size [12].

A cadmium caprylate melt, which forms a mesophase in the temperature range $98-165^{\circ} \mathrm{C}$ and has reducing properties, was chosen as medium for the synthesis of gold, silver and copper nanoparticles. Nanocomposites formed by a vitrified mesophase $\mathrm{Cd}\left(\mathrm{C}_{7} \mathrm{H}_{15} \mathrm{COO}\right)_{2}$ in which metal nanoparticles had been stabilized, were investigated by polarization microscopy. The texture in polarized light was the same as that of the mesophase of pure $\mathrm{Cd}\left(\mathrm{C}_{7} \mathrm{H}_{15} \mathrm{COO}\right)_{2}$, i.e. it had the appearance typical of smectic A with fan-shaped or focal conic texture (Fig. 1). Because of the strong tendency to homeotropic orientation of the LC domains and their small size, the observed texture was fine grained both for the pure mesophase $\mathrm{Cd}\left(\mathrm{C}_{7} \mathrm{H}_{15} \mathrm{COO}\right)_{2}$ and for the mixture with metal nanocrystals (Fig. 1).

Glassy nanocomposites with 4 mol.\% metal nanoparticles were investigated by electronic absorption spectroscopy. It was found that the positions of the absorption peaks of surface plasmon resonance (SPR) for gold, silver and copper nanoparticles were $\lambda=540 \mathrm{~nm}, \lambda=427 \mathrm{~nm}$ and $\lambda=575 \mathrm{~nm}$, respectively, in agreement with literature data. This indicates predominant formation of nanoparticles with a size of $25 \mathrm{~nm}$, $15 \mathrm{~nm}$, and $5 \mathrm{~nm}$ for gold, silver, and copper, respectively $[19,20]$.

Optimization of the synthesis conditions was performed in the work. It was found that the temperature of the melt affects the duration of the reduction process of the gold and silver ions, as well as their size distribution. For instance, increasing the average synthesis temperature from $120^{\circ} \mathrm{C}$ to $180^{\circ} \mathrm{C}$ reduces the average synthesis time from $6 \mathrm{~h}$ to $1 \mathrm{~h}$. At $180^{\circ} \mathrm{C}$, cadmium caprylate changes from liquidcrystalline to an isotropic melt, which results in the formation of larger $(>20 \mathrm{~nm})$ nanoparticles. The liquid-crystalline ordering of the medium for the synthesis of silver nanoparticles permits obtaining small-sized $(<20 \mathrm{~nm})$ silver nanoparticles. In the continuation of our research we used $120^{\circ} \mathrm{C}$ and $150^{\circ} \mathrm{C}$ as synthesis temperature for gold and silver nanoparticles. It was found that copper nanoparticles are formed in the temperature range $185-200^{\circ} \mathrm{C}$, i.e. at the isotropic melt temperature. As can be seen, the optimal synthesis temperature increases in the order $\mathrm{Au}\left(120^{\circ} \mathrm{C}\right), \mathrm{Ag}\left(150^{\circ} \mathrm{C}\right), \mathrm{Cu}\left(200^{\circ} \mathrm{C}\right)$, and the average nanoparticle size decreases in the same order $(25 \mathrm{~nm}$, $15 \mathrm{~nm}, 5 \mathrm{~nm})$. This may be explained by an enhancement of the interaction between the surface atoms and the liquid-crystalline medium, and by an increase of the metal atom diffusion rate. The latter correlates both with the decrease of the molar mass of the metal ions and with the ratio of the mass of the cation to its radius $\left(M_{\mathrm{r}} / r\right)$, the metal cation radii having rather close values (the data were taken from Shannon [21], taking into consideration the coordination number) (Fig. 2).

Fig. 3 shows the dependence of the optical absorption spectra of the metal nanoparticles on the synthesis time for silver nanoparticles, for, as an example, an initial silver ion concentration of $4 \mathrm{~mol} . \%$ and a synthesis temperature of $150^{\circ} \mathrm{C}$. It is seen from the figure that when silver nanoparticles are formed, a plasmon resonance band appears as a small peak at $\lambda=440 \mathrm{~nm}$ (curve 2). The intensity of the peak increases with increasing synthesis time (curve 3 ). In this case, a change from the transparent colorless melt (curve 1) after the first hour of synthesis, to a melt of yellow (curve 2) and light brown (curve 3) color after two and three hours synthesis, respectively, was visually observed.
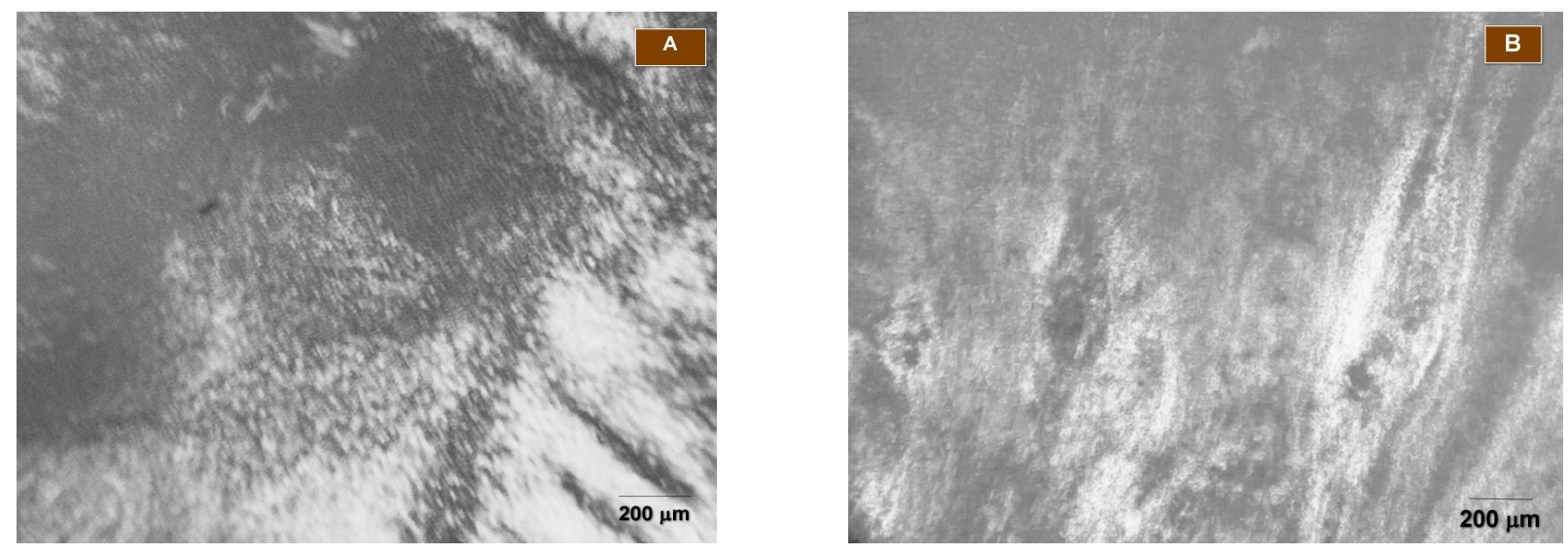

Fig. 1 Textures observed with the aid of a polarizing microscope for pure $\mathrm{Cd}\left(\mathrm{C}_{7} \mathrm{H}_{15} \mathrm{COO}\right)_{2}$ mesophase (a) and a mixture with copper nanoparticles (b) at $150^{\circ} \mathrm{C}$. 


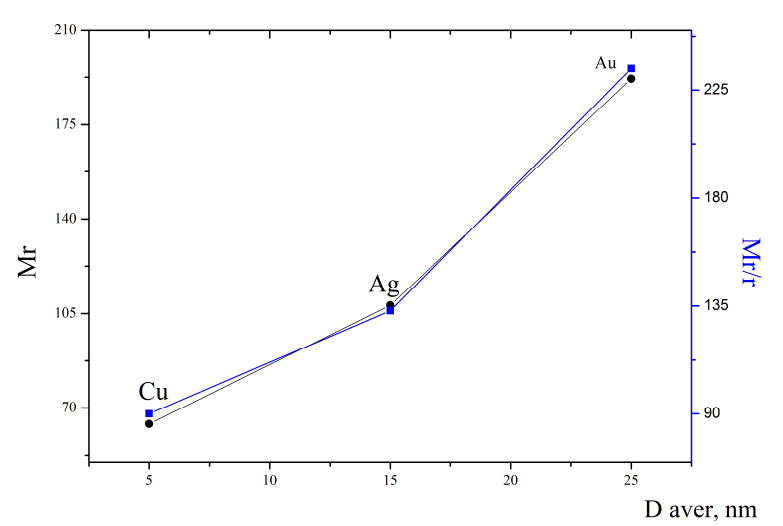

Fig. 2 Dependence of the average size of the obtained metal nanoparticles on the molar mass of the metal $\left(M_{\mathrm{r}}\right)$ (dark circles) and the ratio of the mass to the radius of the cation $\left(M_{\mathrm{r}} / r\right)$ (dark squares) (radii of metal cations according to Shannon [21], taking into consideration the coordination number of the metal cation in the LC alkanoate melt [22]).

The effect of the initial concentration of gold and silver ions on the optical properties of a glassy composite of cadmium caprylate with noble metals nanoparticles was studied. In the case of copper ions, no effect was detected. Increasing the initial concentration of gold ions from 0.25 to $8 \mathrm{~mol} . \%$ and that of silver ions from 0.5 to $8 \mathrm{~mol} . \%$ did practically not affect the synthesis time, but affected the position of the absorption peak of local SPR (Fig. 4). Varying the concentration of gold and silver ions led to an increase of the average nanoparticle size, as indicated by a red shift of the position of the plasmon resonance band maximum from 520 to $550 \mathrm{~nm}$ and from 410 to $440 \mathrm{~nm}$, respectively.

Increasing the amount of both gold and silver nanoparticles in the matrix resulted in a higher effective concentration of metal atoms in the condensed medium. This caused a considerable increase of the nucleation rate and, hence, of the average nanoparticle size. As can be seen, the curve of increase of the average size of the silver nanoparticles is smoother and has no plateau like that of the gold nanoparticles. This may be due to stronger interactions between the silver nanoparticles and the alkanoate medium than for the gold nanoparticles. As seen from Fig. 4, the maximum average size of the gold nanoparticles $(\approx 25 \mathrm{~nm})$ was reached already at $2 \%$. However, despite weak interaction with the alkanoate medium, there is no sharp increase of the size of the gold nanoparticles as a result of the rigid smectic order of the carbon chains of the caprylate matrix.

An estimation of the size of the gold and silver nanoparticles in the nanocomposites based on cadmium caprylate with 4 mol.\% of nanoparticles was performed by transmission electron microscopy.

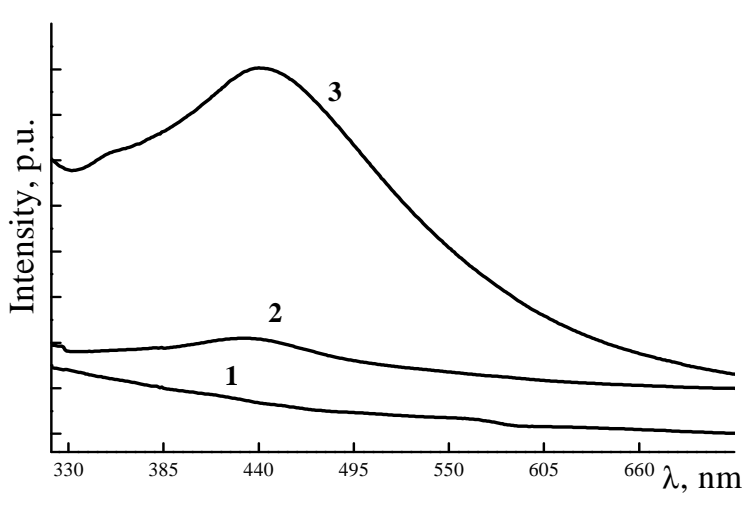

Fig. 3 Absorption spectra of a mesomorphic glassy nanocomposite based on $\mathrm{Cd}\left(\mathrm{C}_{7} \mathrm{H}_{15} \mathrm{COO}\right)_{2}$ with silver nanoparticles for different synthesis times at $150^{\circ} \mathrm{C}$ : (1) 1 hour, (2) 2 hours, (3) 3 hours.

As it is seen from the micrographs (Fig. 5), the Ag and $\mathrm{Au}$ nanoparticles are uniformly distributed throughout the matrix and have spherical shape with a mean diameter of $15 \mathrm{~nm}$ and $25 \mathrm{~nm}$, respectively.

In addition, for the $\mathrm{Au}$ nanoparticles, a size distribution diagram was constructed on the basis of a TEM image (Fig. 6). As indicated above, small-angle $\mathrm{X}$-ray scattering was also used to estimate the average size of the gold nanoparticles (Table 1). Based on these data, the following characteristics were derived: - the electron radius of gyration of the particles, $R_{\mathrm{g}}$, by Guinier extrapolation in the region with $R_{\mathrm{g}}<1.3$; - $R_{\mathrm{g}}$ from the distance distribution function $P(R)$ by the indirect Fourier transform method;

- the volumetric size distribution function of the nanoparticles with allowance for polydispersity of the system.

As is seen from Fig. 6 and Table 1, size polydispersity with an average size of $24-26 \mathrm{~nm}$ is evident for the investigated nanocomposites.

We revealed in this work that the SPR band of gold and silver nanoparticles shifts with increasing metal cation radius and charge of the second component in cadmium caprylate-based binary systems (Fig. 7). The covalent properties of the alkanoate matrix were enhanced in the order $\mathrm{Cd}, \mathrm{Mg}$, $\mathrm{Zn}$. This results in an enhancement of the interaction between the surface atoms and the environment, which leads, as said above, to a decrease of the average nanoparticle size. This manifests itself in the absorption spectra by a blue (towards shorter wavelengths) shift of the positions of the absorption peaks. As seen from Fig. 7, this was observed both for the silver and gold nanoparticles.

As the ionic radius increases in the order $\mathrm{Cd}, \mathrm{Pb}$, and $\mathrm{Li}, \mathrm{Na}, \mathrm{K}$, the ionic strength of the caprylate matrices increases, and the (covalent) properties decrease. On the one hand, this results in weakening 

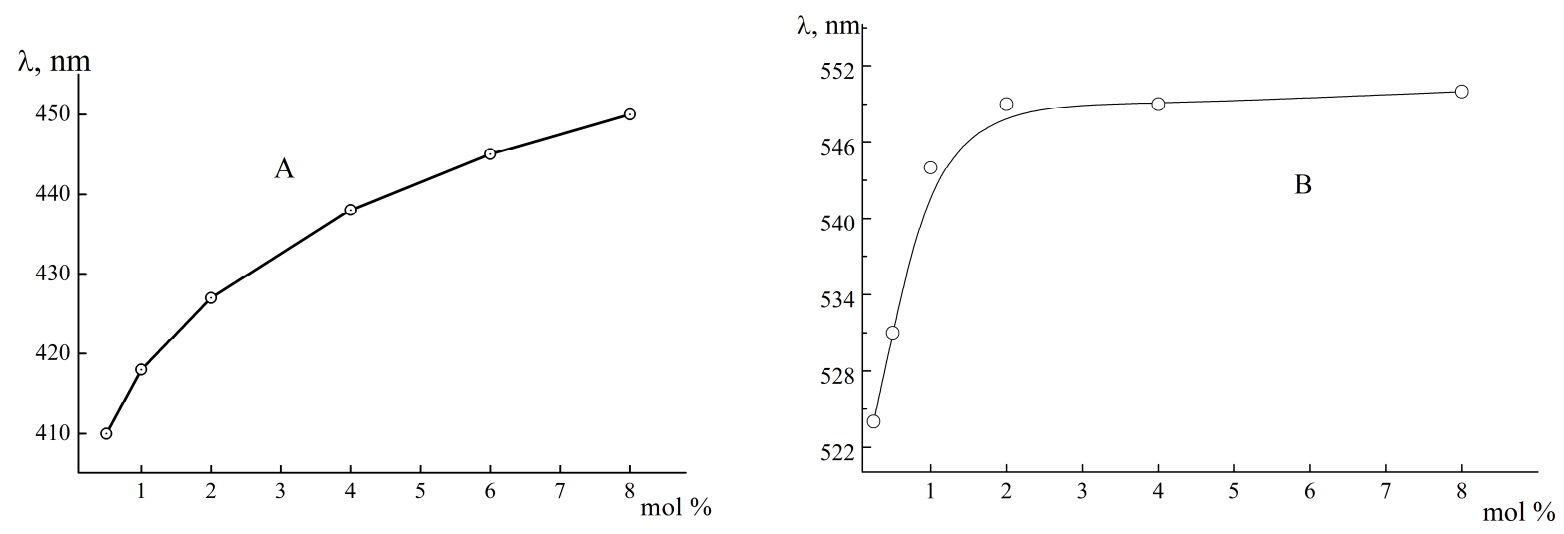

Fig. 4 Dependence of the position of the optical absorption peak of mesomorphic glassy composites based on $\mathrm{Cd}\left(\mathrm{C}_{7} \mathrm{H}_{15} \mathrm{COO}\right)_{2}$ with silver nanoparticles (a) and gold nanoparticles (b) on the concentration of metal nanoparticles; synthesis time $3 \mathrm{~h}$.
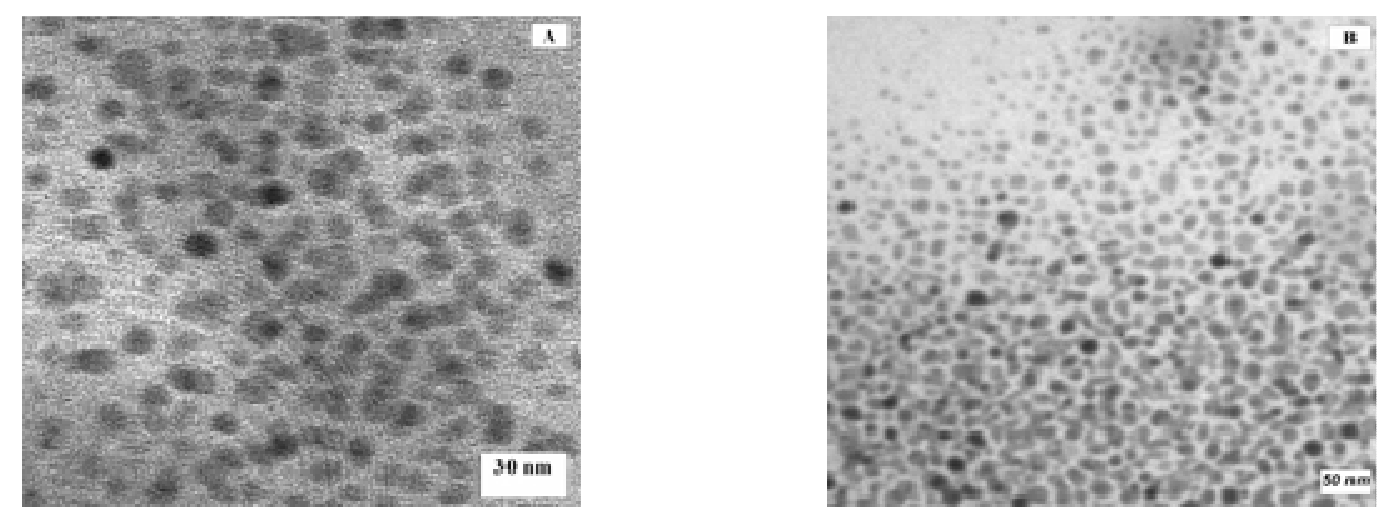

Fig. 5 TEM image of a mesomorphic nanocomposite with silver nanoparticles (a) and gold nanoparticles (b); metal nanoparticle concentration $4 \mathrm{~mol} . \%$, synthesis time $3 \mathrm{~h}$.

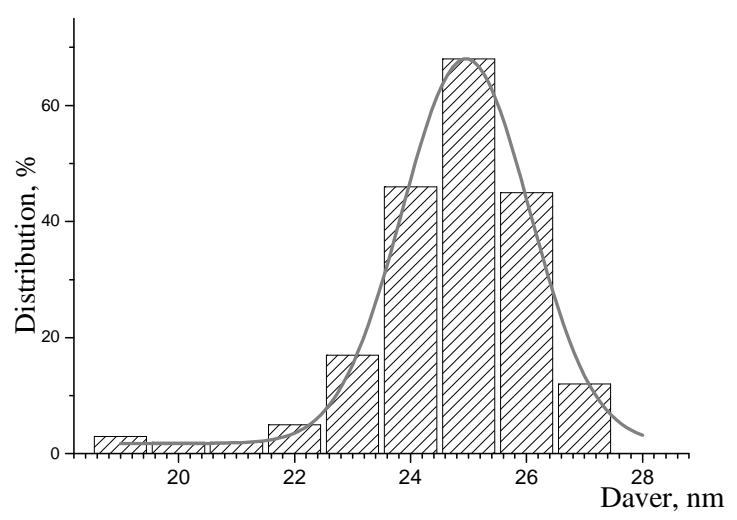

Fig. 6 Size distribution histogram of gold nanoparticles in a mesomorphic glassy cadmium caprylate nanocomposite; gold nanoparticle concentration $4 \mathrm{~mol} . \%$, synthesis time $3 \mathrm{~h}$. of the interaction between the surface atoms and the alkanoate environment, which leads, as said above, to an increase of the average nanoparticle size. On the other hand, the smectic order becomes looser, which results in freer motion of the nanoparticles and in the possibility of reorganization into both larger and smaller nanoparticles. For the silver nanoparticles, the influence of the interaction between nanoparticles and the alkanoate medium predominates. In this case, a shift of the absorption peaks to the region of longer wavelengths of the spectrum takes place, indicating an increase of the average nanoparticle size. As seen from Fig. 7, for the gold nanoparticles an essentially opposite process, namely a shift of the absorption peaks towards the low wavelength region, is observed, indicating a decrease of the average nanoparticle size. In this case, the structuring factor seems to predominate. Since the interaction of the surface atoms of the gold nanoparticles with the alkanoate medium becomes very weak with increasing ionic strength of the caprylate matrix, the kinetic factors of 

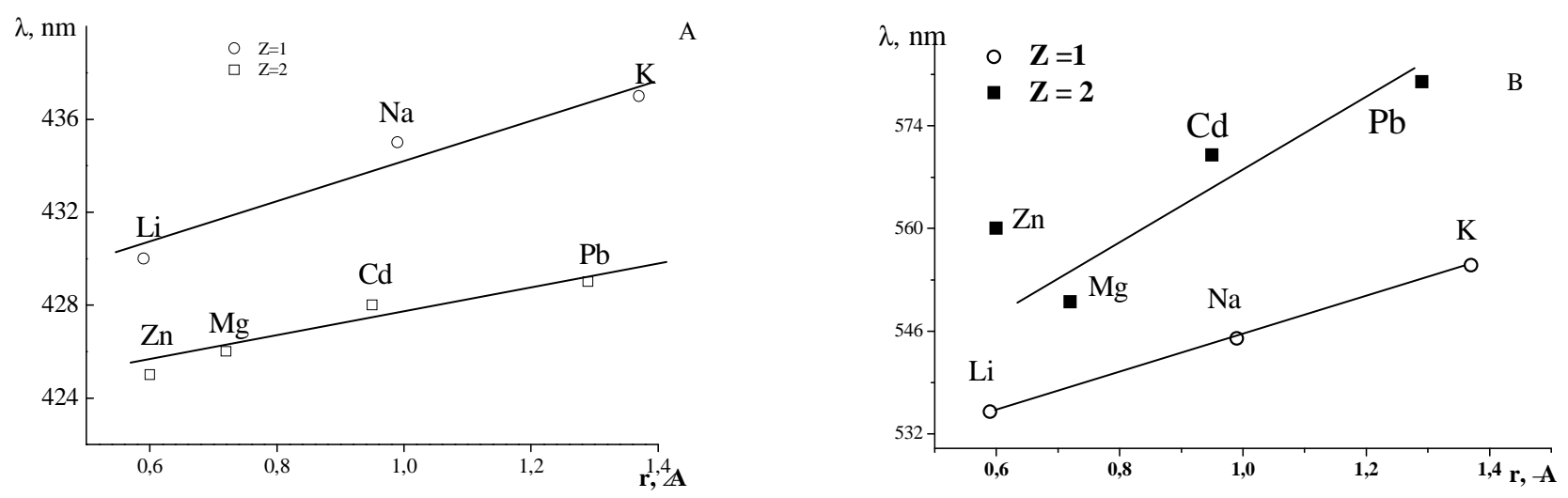

Fig. 7 Dependence of the position of the SPR band maximum of silver (a) and gold (b) nanoparticles on the cation radius and charge of the second component of the LC binary system of equimolar composition $\mathrm{Cd}, M / / \mathrm{C}_{8} \mathrm{H}_{15} \mathrm{O}_{2}$, where $M=\mathrm{Li}, \mathrm{Na}, \mathrm{K}, \mathrm{Mg}, \mathrm{Zn}, \mathrm{Cd}, \mathrm{Pb}$ (radii of metal cations according to Shannon [21] taking into consideration the coordination number of the metal cation in the LC alkanoate melt [22]).

Table 1 Average gold nanoparticle size calculated on the basis of small-angle X-ray scattering data for $\mathrm{Cd}\left(\mathrm{C}_{7} \mathrm{H}_{15} \mathrm{COO}\right)_{2}+4$ mol. $\%$ Au nanocomposite.

\begin{tabular}{c|c}
\hline Calculation technique & Average gold nanoparticle size D $(\mathrm{nm})$ \\
\hline Juinier extrapolation & 24 \\
Indirect Fourier transform method & 28 \\
On the basis of the size distribution function & 15 \\
\hline
\end{tabular}

the nanoparticle growth become of primary importance. The energy barrier for the motion of nanoparticles probably decreases so that the diffusion rate of the gold nanoparticles exceeds their nucleation rate. As a result, the possibility of exchange and rearrangement of nanoparticles increases, and due to the oriented attachment mechanism, a more ordered reorganization of nanoparticles accompanied by a decrease of the average nanoparticle size takes place.

\section{Conclusions}

Gold, silver and copper nanoparticles have been synthesized in melts of cadmium caprylate and solid solutions based on it. Increasing the concentration of the metal ions led to an increase of the average metal nanoparticle size. The metal nanoparticles were uniformly distributed throughout the matrix and had spherical shape.

The influence of different factors (structural and interaction characteristics) of the alkanoate matrix on the formation of nanoparticles was shown. The cationic composition of the LC matrix affects the optical properties of the obtained LC glasses with gold and silver nanoparticles. Depending on the conditions, the position of the maximum absorption of electromagnetic radiation by the surface atoms of the metal nanoparticles shifts either to the long wavelength or to the short wavelength region of the spectrum. This is associated with differences in the cationic composition of the original LC matrices, with the charge and radius of the cations that form part of the LC matrix, and with differences in the nature of the metal nanoparticles.

In conclusion, cadmium caprylate, which forms a nanostructured medium in the melt, allows the creation of novel nanocomposite materials with valuable and controllable optical properties.

\section{References}

[1] N. Toshima, T. Yonezawa, New J. Chem. 22(11) (1998) 1179-1201.

[2] J. Dionne, H. Atwater, MRS Bull. 37(8) (2012) 717-724.

[3] K. Beloglazkina, A. Mazhuga, R. Romashkina, N. Zyk, N. Zefirov, Usp. Khim. 81(1) (2012) 65-90.

[4] A.P. Silva, A.P. Carmo, V. Anjos, M.J.V. Bell, L.R.P. Kassa, R.A. Pinto, Opt. Mater. 34 (2011) 239-243.

[5] F. Chen, S. Dai, T. Xu, X. Shen, C. Lin, Q. Nie, C. Liu, J. Heo, Chem. Phys. Lett. 514 (2011) 79-82.

[6] D. Ray, V.K. Aswal, J. Kohlbrecher, Langmuir 27(7) (2011) 4048-4056.

[7] Yu.A. Krutyakov, A.A. Kudrinskii, A.Yu. Olenin, J.V. Lisichkin, Usp. Khim. 77(3) (2008) 242-269. 
[8] S. Bolisetty, J.J. Vallooran, J. Adamcik, S. Handschin, F. Gramm, R. Mezzenga, J. Colloid Interface Sci. 361 (2011) 90-96.

[9] T.A. Mirnaya, V.N. Asaula, S.V. Volkov, A.S. Tolochko, G.V. Klimusheva, Fiz. Khim. Tverd. Tila 13(1) (2012) 131-136.

[10] V.N. Asaula, T.A. Mirnaya, J.J. Yaremchuk, A.S. Tolochko, Ukr. Khim. Zh. 77(1) (2011) 24-27.

[11] P. Konarev, M. Petoukhov, V. Volkov, D. Svergun, J. Appl. Crystallogr. 39 (2006) 277-286.

[12] A.Yu. Olenin, G.V. Lisichkin, Usp. Khim. 80(7) (2011) 635-662.

[13] O.S. Oluwafemi, N. Revaprasadu, O.O. Adeyemi, Colloids Surf., B 79 (2010) 126-130.

[14] J. Akhtar, M.M. Azad, P. O'Brien, N. Revaprasadu, Mater. Lett. 77 (2012) 78-81.

[15] L. Sun, W. Luan, Yu. Shan, S. Tu, Chem. Eng. J. 189-190 (2011) 451-455.
[16] A. Knauer, A. Thete, H. Romanus, A. Csaki, J.M. Kohler, Chem. Eng. J. 166 (2011) 1164-1169.

[17] E. Dertli, E.N. Esenturk, J. Mater. Res. 28(02) (2013) 250-260.

[18] E.M. Soldatenko, S.Yu. Doronin, R.K. Chernova, Butlerov Commun. 37(2) (2014) 103-113 (in Russian).

[19] M.G. Ventura, A.J. Parola, A. Pires de Matos, J. Non-Cryst. Solids 357(4) (2011) 1342-1349.

[20] M.P. Pileni, J. Phys. Chem. 97 (1993) 6961-6973.

[21] R.D. Shannon, Acta Crystallogr. A 32(5) (1976) 751-767.

[22] T.A. Mirnaya, S.V. Volkov, In: R.D. Rogers, K.R. Seddon, S.V. Volkov (Eds.), Green Industrial Applications of Ionic Liquids, NATO Science Series II, vol. 92, Kluwer Academic Publishers, Dordrecht-Boston-London, 2002, pp. 439-456. 